\title{
Fault Detection for Fuzzy Systems with Multiplicative Noises under Periodic Communication Protocols
}

\author{
Yuqiang Luo, Zidong Wang, Fellow, IEEE, and Guoliang Wei
}

\begin{abstract}
This paper is concerned with the fault detection problem for a class of networked fuzzy systems with multiplicative noises on both system states and measurement outputs. In view of the limited communication capacity, a periodical communication protocol (i.e. the Round-Robin protocol) is adopted to undertake the transmission task between the sensors and the fault detection filter, which leads to periodical delays in the overall system. A Takagi-Sugeno (T-S) fuzzy- model-based fault detection filter is constructed to produce the residual signal and an auxiliary error system is established to facilitate the stability analysis of the error dynamics. With the aid of Lyapunov stability theory, sufficient conditions are obtained that ensure the exponentially mean-square stability of the error dynamics with prescribed $H_{\infty}$ performance constraint. The desired fault detection filter is designed by solving a convex optimization problem via the semi-definite programme method. A finite-time evaluation function and an adjustable threshold are introduced in order to detect the possible faults effectively. The effectiveness of the proposed fault detection scheme is validated by a numerical simulation example.
\end{abstract}

Index Terms-Networked system, fuzzy model, multiplicative noises, Round-Robin protocol, periodical communication, fault detection, residual evaluation function, adjustable threshold.

\section{INTRODUCTION}

In the past two decades, networked control systems (NCSs) have become more and more popular in industrial applications due to their distinct advantages such as flexible structure, far transfer distance, decentralization function, and simple installation. Accordingly, a rich body of literature has been available on various network-induced phenomena including packet dropouts, communication delays, signal quantizations and network congestions, see e.g. [2], [7], [15], [17], [21], [27], [36], [40] and the references therein. A particular cause of network-induced side effect of almost all NCSs is the limited network bandwidth which, to a great extent, prevents the system components (e.g. sensors, actuators and receivers) from hurdle-free communications. Accordingly, significant research attention has been devoted to the investigation on NCSs under

This work was supported in part by the Royal Society of the UK, the National Natural Science Foundation of China under Grants 61329301 and 61374039, the Hujiang Foundation of China under Grants C14002 and D15009, and the Alexander von Humboldt Foundation of Germany.

Y. Luo and G. Wei are with Shanghai Key Lab of Modern Optical System, Department of Control Science and Engineering, University of Shanghai for Science and Technology, Shanghai 200093, China. (Email: guoliang.wei1973@gmail.com)

Z. Wang is with the Department of Computer Science, Brunel University London, Uxbridge, Middlesex, UB8 3PH, United Kingdom. (Email: Zidong.Wang@brunel.ac.uk) limited communication capacity, see e.g. [5], [6], [11], [30], [38]. Among others, the so-called Round-Robin protocol has been widely recognized as an effective means in tackling the communication constraints. The main idea of the Round-Robin scheduling is to assign the transmission opportunity to the communication nodes in a periodic order, thereby dramatically reducing the communication costs. Very recently, the RoundRobin protocol in the network environment has generated considerable research interest from both academy and industry. For instance, a Round-Robin interconnection rule has been designed for a class of distributed observer networks in [35]. In [18], [43], based upon the periodic scheduling mechanism, analysis and synthesis issues of NCSs with Round-Robin protocol have been thoroughly researched by using the timedelay system approach.

As is well known, system faults may degrade the system performance and even cause instability/oscillations leading to cascaded disasters, and fault detection (FD) has proven to be an important area of research with successful applications in a variety of engineering practice [41], [45], [46]. Generally speaking, the first step of model-based FD to design a FD filter to produce a valuable residual signal, and then the second step is to formulate a residual evaluation function to compare with a prescribed threshold. In the past decades, a multitude of FD techniques have been developed and a great number of FD results have been available, see e.g. [20], [24], [25], [44]. In the context of networked systems (e.g. distributed control systems, unattended monitoring systems, remote medical platforms), the FD problem becomes even more critical since the faults might occur more frequently in NCS and seriously jeopardize the system reliability of systems [13]. In [39], the fault detection filtering has been investigated for complex systems with nonhomogeneous Markovian parameters, where the malicious packet losses over communication networks have been considered. On the other hand, an appropriate use of the threshold plays a key role in determining the detection rate and the false alarm rate. Traditionally, the threshold has been chosen as the supremum of the evaluation function in the faultfree case. Such threshold is typically a constant that does not allow adjustment to adapt the changing environment. Recently, the design problem of adaptive thresholds has started to draw some initial research attention. For example, in [1], [20], an adaptive threshold has been studied for the health monitoring of offshore wind-farms and nonlinear systems, respectively.

On another research frontier, for decades, the control and filtering problems for fuzzy systems have been attracting an 
ever-increasing research interest. The fuzzy-logic theory has demonstrated its great superiority in the research of nonlinear systems since Zadeh first proposed the fuzzy set theory. Among various fuzzy approximation models, the Takagi-Sugeno (T-S) fuzzy model is one of the most widely used approaches for studying analysis and synthesis problems of affine nonlinear systems [4]. Under the framework of T-S fuzzy model, the nonlinear plant can be approximated by a set of linear model through local linearization method, where these linear subsystems are smoothly connected by some nonlinear fuzzy membership functions. After tens of years' advances, a large amount of literature have appeared on the analysis/synthesis problems for various nonlinear systems by using the T-S fuzzy model, see e.g. [10], [16], [26], [28], [29], [34], [40] for some representative works. Due to its advantages such as simple installation and maintenance, reduced weight and power requirements, high reliability, etc., the fuzzy networked nonlinear systems have gained many research attention in recent years, see e.g. [10], [27]. When it comes to the fault detection problem, the fuzzy-logic-based approach gives rise to the adaptive nature of the fault detection process [31], [32]. The design of the T-S fuzzy model based fault detector can be easily retrieved in the literature, see e.g. [8], [19], [48].

Summarizing the discussions made so far, it can be concluded that 1) the design of NCSs has become a research focus and the periodical communication protocol (i.e. the RoundRobin protocol) has made it possible to reduce communication burden when the network resource is a concern; 2) the FD problem for NCSs has been well studied and the adaptive threshold selection issue remains open especially in a networked environment; and 3) the fuzzy-model-based analysis approach has proven to be a popular tool in handling nonlinear systems. To this end, a seemingly natural research problem is whether/how we can deal with the FD problem for NCSs under periodical communication protocol. Obviously, such a problem is of both the theoretical importance and practical significance. A thorough literature review reveals that, so far, there have been very few research results on the fault detection problem for networked systems under communication protocols, not to mention the case where the T-S fuzzy model is used as an approximation of certain nonlinear systems as well as the case where the underlying system is subject to multiplicative noises that occur frequently in practice [22]. It is, therefore, the main purpose of this paper to shorten such a gap.

In this paper, we are interested in the fault detection problem for a class of fuzzy networked systems where the desired fault detection filter is implemented under a kind of periodical communication protocol. The contributions of this paper are highlighted as follows: 1) a periodical Round-Robin protocol is introduced to tackle the communication constraints, under which the sensor measurement at the receiving end is presented; 2) the multiplicative noises on both the system states and the measurement outputs are taken into account to describe the interference from a non-ideal environment; 3 ) an $H_{\infty}$ fault detection filter is constructed on the basis of the T-S fuzzy model such that the detection error dynamics is exponentially stable in the mean square sense; 4) the difficulty (mainly periodic time-delays) resulting from the combinational use of Round-Robin protocol and zero-order-holders (ZOHs) is effectively overcome by developing the block matrix technique; and 5) an adjustable threshold is suggested that provides more flexibility for the detector design as compared to the traditional case of constant threshold.

Notation. In this paper, $\mathbb{R}^{n}, \mathbb{R}^{n \times m}$ and $\mathbb{Z}\left(\mathbb{Z}^{+}, \mathbb{Z}^{-}\right)$denote, respectively, the $n$-dimensional Euclidean space, the set of all $n \times m$ real matrices and the set of all integers (nonnegative integers, negative integers). $\|\cdot\|$ refers to the Euclidean norm in $\mathbb{R}^{n} . I_{n}$ represents the identity matrix of dimension $n \times n$, and $I$ is the identity matrix of compatible dimension. The notation $X \geq Y$ (respectively, $X>Y$ ), where $X$ and $Y$ are symmetric matrices, means that $X-Y$ is positive semi-definite (respectively, positive definite). For a matrix $M, M^{T}$ and $M^{-1}$ represent its transpose and inverse, respectively. The shorthand $\operatorname{diag}\left\{M_{1}, M_{2}, \ldots, M_{n}\right\}$ denotes a block diagonal matrix with diagonal blocks being the matrices $M_{1}, M_{2}, \ldots, M_{n}$. In symmetric block matrices, the symbol ' $*$ ' is used as an ellipsis for terms induced by symmetry. $\lambda_{\max }(\cdot)$ is the maximum eigenvalue. $\bmod (a, b)$ represents the unique nonnegative remainder on division of the integer $a$ by the positive integer $b$. For integers $a, b$ with $a \leq b, \mathbb{N}[a, b]$ denotes the discrete interval given by $\mathbb{N}[a, b]=[a, a+1, \cdots, b-1, b]$. Matrices, if they are not explicitly stated, are assumed to have compatible dimensions.

\section{PRoblem Formulation}

Consider the following discrete networked fuzzy systems with multiplicative noises:

\section{Plant Rule $i$ :}

IF $\theta_{1}(k)$ is $\mathcal{F}_{i 1}, \cdots, \theta_{j}(k)$ is $\mathcal{F}_{i j}, \cdots$ and $\theta_{p}(k)$ is $\mathcal{F}_{i p}$, THEN

$$
\left\{\begin{aligned}
x(k+1)= & \left(A_{i}+\zeta(k) D_{i}\right) x(k)+B_{i} u(k) \\
& +E_{i} \omega(k)+F_{i} f(k), \\
y(k)= & \left(C_{i}+\xi(k) G_{i}\right) x(k)+H_{i} \omega(k), \quad i \in \mathbb{U} \\
x(k)= & \phi(k), \quad \forall k \in \mathbb{Z}^{-}
\end{aligned}\right.
$$

where $k \in \mathbb{Z}^{+}, \mathbb{U}=\{1,2, \cdots, r\}$ with $r$ being the number of IF-THEN rules; $\theta(k)=\left[\theta_{1}(k), \theta_{2}(k) \cdots, \theta_{p}(k)\right]$ is the premise variable vector; $\mathcal{F}_{i j}(j=1,2, \cdots, p)$ is the fuzzy set; $x(k) \in \mathbb{R}^{n}$ is the state vector; $u(k) \in \mathbb{R}^{n_{u}}$ is the control input vector; $y(k)=\left[y_{1}(k), y_{2}(k), \cdots, y_{l}(k), \cdots, y_{s}(k)\right]^{T}$ is the measured output vector with $y_{l}(k) \in \mathbb{R}$ being the $l$ th sensor measurement $(l \in \mathbb{S}=\{1,2, \cdots, s\}) ; \phi(k)$ is the initial state; $\zeta(k) \in \mathbb{R}$ and $\xi(k) \in \mathbb{R}$ are the zero-mean multiplicative noises satisfying $\mathbb{E}\left\{\zeta^{2}(k)\right\}=\sigma_{\zeta}^{2}$ and $\mathbb{E}\left\{\xi^{2}(k)\right\}=\sigma_{\xi}^{2}$, respectively; $\omega(k) \in \mathbb{R}^{n_{\omega}}$ is the unknown disturbance input which belongs to $l_{2}[0, N)$ (the space of square summable sequences with the norm of $\|\omega\|_{[0, N]}^{2}=\mathbb{E}\left\{\sum_{k=0}^{N}\|\omega(k)\|^{2}\right\}$ [33]); $f(k) \in \mathbb{R}^{n_{f}}$ is the possible fault signal to be detected; and $A_{i}, D_{i}, B_{i}, E_{i}, F_{i}, C_{i}, G_{i}$ and $H_{i}$ are constant matrices with appropriate dimensions.

Remark 1. As is well known, the system model (1) is actually the local linear models that can be used to approximate a nonlinear plant at any precision through the nonlinear fuzzy membership functions. On the other hand, the multiplicative 
noises not only enter the system states but also appear in the measured outputs. Therefore, the concerned model is closed to engineering practice [42]. In this paper, for simplicity, the second-order statistics of the multiplicative noises is assumed to be known, and $\zeta(k)$ and $\xi(k)$ are uncorrelated with each other.

In this paper, we assume that the sensors are distributed in different locations from the target networked fuzzy system, and the communication is conducted through shared networks. In order to mitigate the negative influence from the limited communication capacity of the communication network, the Round-Robin protocol is utilized as the communication protocol to schedule the sensors. The so-called Round-Robin protocol can be described by the following rules:

$i$ ) all sensors are pre-arranged in a round sequence as the communication nodes;

ii) all communication nodes are orderly allowed to access the channel at a time instant $k$;

iii) each communication node can only take one unit time of the index $k$ for one communication link.

For illustration purpose, the channel access order of sensors under the Round-Robin protocol is shown in Fig. 1, in which the channel accesses of each sensor node are divided into different round. From the mechanism of Round-Robin scheduling, it can be known that the $l$ th sensor measurement $y_{l}(k)$ can only be transmitted at the time instant $k=s j+l$ $\left(j \in \mathbb{Z}^{+}\right)$. Therefore, a sensor's access to the channel is periodic. On the other hand, in order to maximize the utilization of the received measurements, a set of $\mathrm{ZOHs}$ are adopted to store the received sensor measurements, where the data will not be updated until the next renewed measurement arrives.

Denoting $\vec{y}_{l}(k)$ as the $l$ th sensor measurement at the receiving end, it is verified that

$$
\vec{y}_{l}(k)=\left\{\begin{array}{cc}
y_{l}\left(k-\aleph_{k}^{l}\right), & k-l \geq 0 \\
0, & k-l<0
\end{array}\right.
$$

where $\aleph_{k}^{l}=\bmod (k-l, s)$ is the time-delay induced by the adopted communication protocol and ZOHs. It is obvious that the delay $\aleph_{k}^{l} \in \Pi_{0}$ appears periodically, where $\Pi_{0}=$ $\{0,1,2, \cdots, s-1\}$.

Remark 2. The Round-Robin protocol serves as an effective method to alleviate the communication burden in network communications with a limited transmission rate. Because of its economic utilization of network bandwidth, the RoundRobin protocol has found wide applications in networked control systems. Moreover, as pointed out in [11], the periodic scheduling method plays an important role in distributed systems (e.g. the microactuator arrays) since the simultaneous communication with multiple subsystems can hardly be achieved. It should be noticed that the preserved measurements in the ZOHs can provide the fault detection filter with full sensor measurements, which are actually the measurement vector $\vec{y}(k)$. Unfortunately, the combinational use of the Round-Robin protocol and the $\mathrm{ZOH}$ sould inevitably introduce certain time-varying periodic delays to the received measurements, which will certainly increase the complexity of the design process. Such kind of periodic delays, if not properly handled, would degrade the performance of the fault detector to some extent [12].

For generating the residual to detect the possible fault in the networked systems, we construct a fuzzy fault detection filter of the following type (which is also called the residual generating system):

Fault detection filter $i$ :

IF $\theta_{1}$ is $\mathcal{F}_{i 1}, \cdots, \theta_{j}$ is $\mathcal{F}_{i j}, \cdots$ and $\theta_{p}$ is $\mathcal{F}_{i p}$, THEN

$$
\left\{\begin{aligned}
\hat{x}(k+1) & =\hat{A}_{i} \hat{x}(k)+\hat{B}_{i} \vec{y}(k), \\
\hat{y}(k) & =\hat{C}_{i} \hat{x}(k)+\hat{D}_{i} \vec{y}(k), \\
r(k) & =\hat{y}(k)-f(k),
\end{aligned}\right.
$$

where $\hat{x}(k) \in \mathbb{R}^{n}$ is the state of the fault detection filter; $\hat{y}(k) \in \mathbb{R}^{n_{f}}$ is the output of the residual generating system; $r(k)$ is the so-called the residual signal; and $\hat{A}_{i}, \hat{B}_{i}, \hat{C}_{i}$ and $\hat{D}_{i}$ are the gain parameters of the concerned fault detection filter to be designed.

For brevity, in what follows, we denote $h_{i}=h_{i}(k)$ and

$$
\begin{aligned}
& \sum_{i_{1}, i_{2}, \cdots, i_{s}=1}^{r} h_{i_{1}} h_{i_{2}} \cdots h_{i_{s}} \\
= & \sum_{i_{1}=1}^{r} h_{i_{1}} \sum_{i_{2}=1}^{r} h_{i_{2}} \cdots \sum_{i_{s}=1}^{r} h_{i_{s}} \quad \text { for } s \in \mathbb{Z}^{+} .
\end{aligned}
$$

Define the normalized membership function (also called the fuzzy basis function) as

$$
h_{i}(k)=\frac{\Psi_{i}(k)}{\sum_{j=1}^{r} \Psi_{j}(k)}
$$

where $\Psi_{i}(k)=\prod_{j=1}^{p} \mathfrak{F}_{i j}\left(\theta_{j}(k)\right)$ and $\mathfrak{F}_{i j}\left(\theta_{j}(k)\right)>0$ is the grade of membership of $\theta_{j}(k)$ in $\mathcal{F}_{i j}$. Apparently, we have

$$
0 \leq h_{i}(k) \leq 1, \quad \sum_{i=1}^{r} h_{i}(k)=1, \quad \forall k \in \mathbb{Z}^{+}
$$

Consequently, the defuzzified output of the networked T-S fuzzy model (1) can be presented as

$$
\left\{\begin{aligned}
x(k+1)= & \sum_{i=1}^{r} h_{i}(k)\left[\left(A_{i}+\zeta(k) D_{i}\right) x(k)+B_{i} u(k)\right. \\
& \left.+E_{i} \omega(k)+F_{i} f(k)\right], \quad i \in \mathbb{U} . \\
y(k)= & \sum_{i=1}^{r} h_{i}(k)\left[\left(C_{i}+\xi(k) G_{i}\right) x(k)+H_{i} \omega(k)\right]
\end{aligned}\right.
$$

By denoting $\hat{B}_{i}=\left[\begin{array}{ll}\hat{B}_{i .1} & \hat{B}_{i .2} \cdots \hat{B}_{i . s}\end{array}\right], \quad \hat{D}_{i}=$ $\left[\begin{array}{ll}\hat{D}_{i .1} & \hat{D}_{i .2} \cdots \hat{D}_{i . s}\end{array}\right]$

$C_{i}=\left[\begin{array}{c}C_{i \circ 1} \\ C_{i \circ 2} \\ \ldots \\ C_{i \circ 5}\end{array}\right], \quad G_{i}=\left[\begin{array}{c}G_{i \circ 1} \\ G_{i \circ 2} \\ \ldots \\ G_{i \circ 5}\end{array}\right], \quad H_{i}=\left[\begin{array}{c}H_{i \circ 1} \\ H_{i \circ 2} \\ \ldots \\ H_{i \circ 5}\end{array}\right]$, 


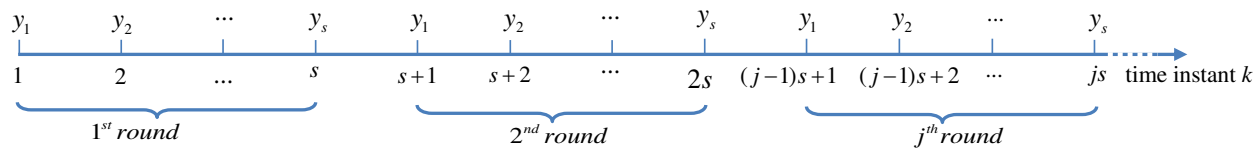

Fig. 1: Access order of sensors under Round-Robin protocol

the fault detection filter (3) can be described by

$$
\left\{\begin{aligned}
\hat{x}(k+1)= & \frac{1}{s} \sum_{i, j=1}^{r} h_{i} h_{j} \sum_{l=1}^{s}\left[\hat{A}_{i} \hat{x}(k)\right. \\
& +s\left(\hat{B}_{i . l} C_{j \circ l} x\left(k-\aleph_{k}^{l}\right)\right. \\
& +\hat{B}_{i . l} G_{j \circ l} \xi\left(k-\aleph_{k}^{l}\right) x\left(k-\aleph_{k}^{l}\right) \\
& \left.\left.+\hat{B}_{i . l} H_{j \circ l} \omega\left(k-\aleph_{k}^{l}\right)\right)\right] \\
\hat{y}(k)= & \frac{1}{s} \sum_{i, j=1}^{r} h_{i} h_{j} \sum_{l=1}^{s}\left[\hat{C}_{i} \hat{x}(k)\right. \\
& +s\left(\hat{D}_{i . l} C_{j \circ l} x\left(k-\aleph_{k}^{l}\right)\right. \\
& +\hat{D}_{i . l} G_{j \circ l} \xi\left(k-\aleph_{k}^{l}\right) x\left(k-\aleph_{k}^{l}\right) \\
& \left.\left.+\hat{D}_{i . l} H_{j \circ l} \omega\left(k-\aleph_{k}^{l}\right)\right)\right]
\end{aligned}\right.
$$

Denoting $\tilde{x}(k)=\hat{x}^{T}(k)-x^{T}(k), \theta(k)=\left[\begin{array}{ll}x^{T}(k) & \tilde{x}(k)\end{array}\right]^{T}$ and $\nu^{T}(k)=\left[\begin{array}{lll}\omega^{T}(k) & f^{T}(k) & u^{T}(k)\end{array}\right]$, we immediately obtain the following augmented error dynamics of the fault detection filter:

$$
\left\{\begin{aligned}
\theta(k+1)= & \frac{1}{s} \sum_{i, j, t=1}^{r} h_{i} h_{j} h_{t} \sum_{l=1}^{s}\left[\bar{A}_{i j t} \theta(k)\right. \\
& +\zeta(k) \bar{B}_{j t} \theta(k)+\bar{C}_{i j l} \theta\left(k-\aleph_{k}^{l}\right) \\
& +\xi\left(k-\aleph_{k}^{l}\right) \bar{D}_{i j l} \theta\left(k-\aleph_{k}^{l}\right) \\
& \left.+\bar{E}_{j t} \nu(k)+\bar{F}_{i j l} \nu\left(k-\aleph_{k}^{l}\right)\right] \\
r(k)= & \frac{1}{s} \sum_{i, j, t=1}^{r} h_{i} h_{j} h_{t} \sum_{l=1}^{s}\left[\bar{G}_{i} \theta(k)\right. \\
& +\bar{H}_{i j l} \theta\left(k-\aleph_{k}^{l}\right) \\
& +\xi\left(k-\aleph_{k}^{l}\right) \bar{J}_{i j l} \theta\left(k-\aleph_{k}^{l}\right) \\
& \left.+K \nu(k)+\bar{L}_{i j l} \nu\left(k-\aleph_{k}^{l}\right)\right]
\end{aligned}\right.
$$

where $K=\left[\begin{array}{ll}0 & -I_{n_{f}}\end{array}\right]$,

$$
\begin{aligned}
& \bar{A}_{i j t}=\left[\begin{array}{cc}
A_{t} & 0 \\
\hat{A}_{i}-A_{j} & \hat{A}_{i}
\end{array}\right], \quad \bar{C}_{i j l}=\left[\begin{array}{cc}
0 & 0 \\
s \hat{B}_{i . l} C_{j o l} & 0
\end{array}\right], \\
& \bar{B}_{j t}=\left[\begin{array}{cc}
D_{t} & 0 \\
-D_{j} & 0
\end{array}\right], \quad \bar{E}_{j t}=\left[\begin{array}{ccc}
E_{t} & F_{t} & B_{t} \\
-E_{j} & -F_{j} & -B_{j}
\end{array}\right], \\
& \bar{D}_{i j l}=\left[\begin{array}{cc}
0 & 0 \\
s \hat{B}_{i . l} G_{j \circ l} & 0
\end{array}\right], \quad \bar{F}_{i j l}=\left[\begin{array}{cc}
0 & 0 \\
s \hat{B}_{i . l} H_{j o l} & 0
\end{array}\right], \\
& \bar{G}_{i}=\left[\begin{array}{ll}
\hat{C}_{i} & \hat{C}_{i}
\end{array}\right], \quad \bar{H}_{i j l}=\left[\begin{array}{ll}
s \hat{D}_{i . l} C_{j \circ l} & 0
\end{array}\right],
\end{aligned}
$$

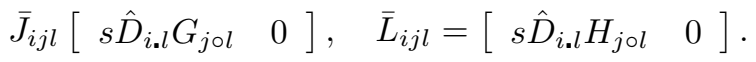

Definition 1. The augmented error dynamics (8) with $\nu(k) \equiv$ 0 is said to be exponentially mean-square stable if there exist two constants $\alpha>0$ and $\epsilon \in(0,1)$ such that

$$
\mathbb{E}\left\{\|\theta(k)\|^{2}\right\} \leq \alpha \epsilon^{k} \sup _{i \in \Pi_{0}} \mathbb{E}\left\{\|\theta(i)\|^{2}\right\}, \quad k \in \mathbb{Z}^{+} .
$$

Definition 2. Under the zero initial condition, if the following disturbance attenuation constraint

$$
\min \gamma \text { s.t. } \sum_{k=0}^{\infty} \mathbb{E}\left\{\|r(k)\|^{2}\right\} \leq \gamma^{2} \sum_{k=0}^{\infty} \mathbb{E}\left\{\|v(k)\|^{2}\right\}
$$

is met for all nonzero sequences $\{v(\cdot)\}$, where $\gamma>0$ and $v(k)=\left[\begin{array}{lll}\nu^{T}(k) & \nu^{T}\left(k-\aleph_{k}^{1}\right) \cdots \nu^{T}\left(k-\aleph_{k}^{l}\right) \cdots \nu^{T}\left(k-\aleph_{k}^{s}\right)\end{array}\right]^{T}$, then the fuzzy residual generating system (3) is regarded as an $H_{\infty}$ fault detection filter.

In this paper, our main purpose is to obtain an $H_{\infty}$ fuzzy fault detection filter in the form of (3) such that the augmented error dynamics (8) is exponentially mean-square stable. Afterwards, based on the residual signal generated by the fuzzy fault detection filter (3), a finite-time residual evaluation function $J(k)$ is established as follows:

$$
J(k)=\mathbb{E}\left\{\left(\sum_{\iota=0}^{\mathcal{L}}\|r(k-\iota)\|\right)^{\frac{1}{2}}\right\},
$$

where $\mathcal{L} \in \mathbb{Z}^{+}$is the length of the evaluating time horizon. In order to determine the time to alarm, a trigger point (i.e. the threshold $J_{t h}$ ) needs to be determined. Among various forms of thresholds in specific implementation, a common choice for the threshold is the supremum of the evaluation function in the fault-free case [14], namely,

$$
\bar{J}_{t h}=\sup _{k \in \mathbb{Z}^{+}, \xi(k) \neq 0, \zeta(k) \neq 0, \omega(k) \in l_{2}, u(k) \neq 0, f(k)=0} \mathbb{E}\{J(k)\} .
$$

However, it is well known that the above threshold inevitably results in a fixed trigger point, and thus cannot be dynamically adjusted. To solve this problem, we adopt a dynamic threshold as follows:

$$
J_{t h}=J_{t h, \omega}+J_{t h, u},
$$

where

$$
\begin{aligned}
& J_{t h, \omega}=\sup _{k \in \mathbb{Z}^{+}, \xi(k) \neq 0, \zeta(k) \neq 0, \omega(k) \in l_{2}, u(k)=0, f(k)=0} \mathbb{E}\{J(k)\}, \\
& J_{t h, u}=\sup _{k \in \mathbb{Z}^{+}, \xi(k) \neq 0, \zeta(k) \neq 0, \omega(k)=0, u(k) \neq 0, f(k)=0} \mathbb{E}\{J(k)\} .
\end{aligned}
$$

As pointed out in [47], the constant $J_{t h, \omega}$ can be determined off-line, but $J_{t h, u}$ can be adjusted on-line by dynamically changing the control input $u(k)$ [47]. Since the residual evaluation function $J(k)$ is influenced by the fault in the target system, the occurred fault can be detected if the value of the finite-time residual evaluation function exceeds the obtained threshold. More specifically, the triggering mechanism of alarm can be described by the following rule:

$$
\begin{aligned}
& J(k)>J_{t h} \Longrightarrow \text { Alarm : fault } \\
& J(k) \leq J_{t h} \Longrightarrow \text { No alarm: fault free. }
\end{aligned}
$$




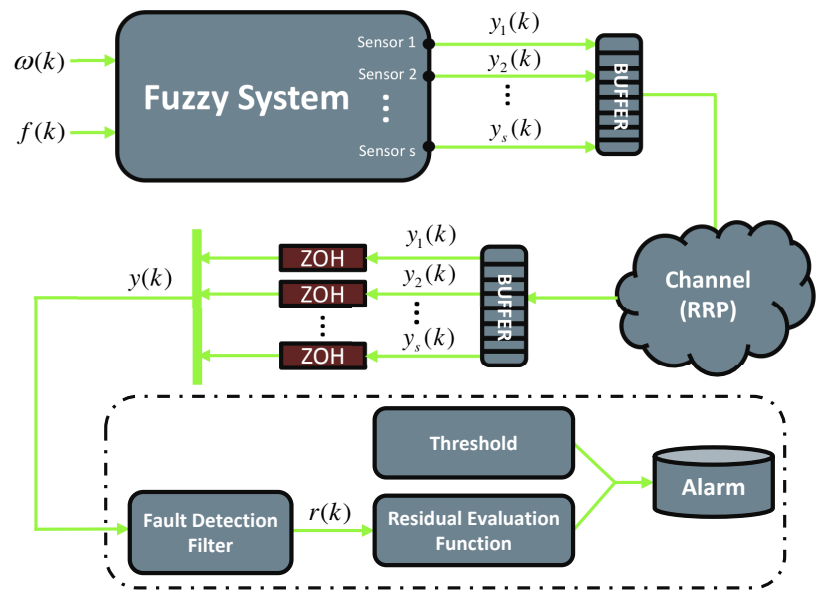

Fault Detector

Fig. 2: Fuzzy fault detection system under Round-Robin protocol

For a better illustration, the flowchart of the fault detection procedure under the Round-Robin protocol is shown in Fig. 2.

\section{MAIN RESUlts}

The following lemmas will be used in the proofs of our main results.

Lemma 1. (Schur Complement) [3] Let the constant matrices $\Sigma_{1}, \Sigma_{2}, \Sigma_{3}$ be given where $\Sigma_{1}=\Sigma_{1}^{T}$ and $0<\Sigma_{2}=\Sigma_{2}^{T}$. Then, $\Sigma_{1}-\Sigma_{3}^{T} \Sigma_{2}^{-1} \Sigma_{3} \geq 0$ if and only if

$$
\left[\begin{array}{cc}
\Sigma_{1} & \Sigma_{3}^{T} \\
\Sigma_{3} & \Sigma_{2}
\end{array}\right] \geq 0 \quad \text { or } \quad\left[\begin{array}{cc}
\Sigma_{2} & \Sigma_{3} \\
\Sigma_{3}^{T} & \Sigma_{1}
\end{array}\right] \geq 0
$$

Lemma 2. Let $R \in \mathbb{R}^{2 n \times 2 n}$ be a positive definite matrix. For any real vectors $X_{i j t l} \in \mathbb{R}^{2 n}$ and $X_{a b c d} \in \mathbb{R}^{2 n}$ with $i, j, t, a, b, c \in \mathbb{U}$ and $l, d \in \mathbb{S}$, we have

$$
\begin{aligned}
& \frac{1}{s} \sum_{i, j, t, a, b, c=1}^{r} h_{i} h_{j} h_{t} h_{a} h_{b} h_{c} \sum_{l=1}^{s} \sum_{d=1}^{s} X_{i j t l}^{T} R X_{a b c d} \\
\leq & \sum_{i, j, t=1}^{r} h_{i} h_{j} h_{t} \sum_{l=1}^{s} X_{i j t l}^{T} R X_{i j t l}
\end{aligned}
$$

where $h_{z} \geq 0$ and $\sum_{z=1}^{r} h_{z}=1$ with $z \in \mathbb{U}$.

Proof: Similar to the proof of Lemma 2 in [9], let us recall the well-known inequality

$$
2 X^{T} R Y \leq X^{T} R X+Y^{T} R Y
$$

where $X$ and $Y$ are any vectors belonging to $\mathbb{R}^{2 n}$. Then, it follows that one easily has

$$
\begin{aligned}
& \frac{2}{s} \sum_{i, j, t, a, b, c=1}^{r} h_{i} h_{j} h_{t} h_{a} h_{b} h_{c} \sum_{l=1}^{s} \sum_{d=1}^{s} X_{i j t l}^{T} R X_{a b c d} \\
\leq & \frac{1}{s} \sum_{i, j, t, a, b, c=1}^{r} h_{i} h_{j} h_{t} h_{a} h_{b} h_{c} \sum_{l=1}^{s} \sum_{d=1}^{s}\left(X_{i j t l}^{T} R X_{i j t l}\right. \\
& \left.+X_{a b c d}^{T} R X_{a b c d}\right) \\
= & 2 \sum_{i, j, t=1}^{r} h_{i} h_{j} h_{t} \sum_{l=1}^{s} X_{i j t l}^{T} R X_{i j t l},
\end{aligned}
$$

which completes the proof.

For the fault detection problem, it is always a prerequisite to generate the residual signal in order to establish the residual evaluation function latter. In what follows, we shall concentrate upon the analysis and synthesis of the proposed fuzzy fault detection filter, where the exponentially mean-square stability of the augmented error dynamics and the $H_{\infty}$ disturbance attenuation performance will be simultaneously addressed.

\section{A. Stability and $H_{\infty}$ performance analysis}

Theorem 1. Let the parameters (i.e., $\hat{A}_{i}, \hat{B}_{i}, \hat{C}_{i}$ and $\hat{D}_{i}$ ) of the fault detection filter be given. The error dynamics (8) is exponentially mean-square stable and the residual-generating system (3) is an $H_{\infty}$ fault detection filter under the RoundRobin scheduling if there exist two positive definite matrices $P$ and $Q$ such that the following semi-definite problem is feasible

$$
\min \gamma \quad \text { s.t. } \bar{\Omega}^{i j t l}<0, \quad i, j, t \in \mathbb{U} \text { and } l \in \mathbb{S}
$$

where $\bar{\Omega}_{3}^{i j t l}=\operatorname{diag}\left\{-Q^{-1},-I_{n_{f}},-Q^{-1},-I_{n_{f}}\right\}$,

$$
\begin{aligned}
\bar{\Omega}^{i j t l} & =\left[\begin{array}{cc}
\bar{\Omega}_{1}^{i j t l} & * \\
\bar{\Omega}_{2}^{i j t l} & \bar{\Omega}_{3}^{i j t l}
\end{array}\right], \quad \Lambda_{1}=Q-s P-\sigma_{\zeta}^{2} \bar{B}_{j t}^{T} Q \bar{B}_{j t}, \\
\bar{\Omega}_{1}^{i j t l} & =\operatorname{diag}\left\{-\Lambda_{1},-s P,-\gamma^{2} I_{\left(n_{\omega}+n_{f}\right)},-s \gamma^{2} I_{\left(n_{\omega}+n_{f}\right)}\right\}, \\
\bar{\Omega}_{2}^{i j t l} & =\left[\begin{array}{cccc}
0 & \sigma_{\xi} \bar{D}_{i j l} & 0 & 0 \\
0 & \sigma_{\xi} \bar{J}_{i j l} & 0 & 0 \\
\bar{A}_{i j t} & \bar{C}_{i j l} & \bar{E}_{j t} & \bar{F}_{i j l} \\
\bar{G}_{i} & \bar{H}_{i j l} & K & \bar{L}_{i j l}
\end{array}\right] .
\end{aligned}
$$

Proof: Define $\Psi_{t}(k)=\{\theta(k), \theta(k-1), \cdots, \theta(k-t)\}$ and $\hbar(k)=\bigcup_{t=1}^{\infty} \Psi_{t}(k)$. Choose a Lyapunov-Krasovskii function candidate as follows

$$
V(k)=\sum_{i=1}^{2} V_{i}(k),
$$

where

$$
\begin{aligned}
& V_{1}(k)=\theta^{T}(k) Q \theta(k), \\
& V_{2}(k)=\sum_{l=1}^{s} \sum_{\tau(k)=-\aleph_{k}^{l}}^{-1} \theta^{T}(k+\tau(k)) P \theta(k+\tau(k)) .
\end{aligned}
$$

Letting $\nu(k)=0$, we compute the difference of $V(k)$ along the trajectory of the error dynamics (8) as follows:

$$
\mathbb{E}\{V(k+1)-V(k) \mid \hbar(k)\}
$$




$$
\begin{aligned}
= & \frac{1}{s^{2}} \sum_{i, j, t, a, b, c=1}^{r} h_{i} h_{j} h_{t} h_{a} h_{b} h_{c} \sum_{l=1}^{s} \sum_{d=1}^{s} \\
& \times \mathbb{E}\left\{\left[\bar{A}_{i j t} \theta(k)+\zeta(k) \bar{B}_{j t} \theta(k)+\bar{C}_{i j l} \theta\left(k-\aleph_{k}^{l}\right)\right.\right. \\
& \left.+\xi\left(k-\aleph_{k}^{l}\right) \bar{D}_{i j l} \theta\left(k-\aleph_{k}^{l}\right)\right]^{T} Q \\
& \times\left[\bar{A}_{a b} \theta(k)+\zeta(k) \bar{B}_{b} \theta(k)+\bar{C}_{a b d} \theta\left(k-\aleph_{k}^{c}\right)\right. \\
& \left.\left.+\xi\left(k-\aleph_{k}^{c}\right) \bar{D}_{a b d} \theta\left(k-\aleph_{k}^{c}\right)\right]-\theta^{T}(k) Q \theta(k)\right\} \\
& +\sum_{l=1}^{s} \mathbb{E}\left\{\theta^{T}(k) P \theta(k)-\theta^{T}\left(k-\aleph_{k}^{l}\right) P \theta\left(k-\aleph_{k}^{l}\right)\right\} \\
= & \frac{1}{s^{2}} \sum_{i, j, t, a, b, c=1}^{r} h_{i} h_{j} h_{t} h_{a} h_{b} h_{c} \sum_{l=1}^{s} \sum_{d=1}^{s} \\
& \times \mathbb{E}\left\{\left[\bar{A}_{i j t} \theta(k)+\zeta(k) \bar{B}_{j t} \theta(k)+\bar{C}_{i j l} \theta\left(k-\aleph_{k}^{l}\right)\right.\right. \\
& \left.+\xi\left(k-\aleph_{k}^{l}\right) \bar{D}_{i j l} \theta\left(k-\aleph_{k}^{l}\right)\right]^{T} Q\left[\bar{A}_{a b} \theta(k)\right. \\
& +\zeta(k) \bar{B}_{b} \theta(k)+\bar{C}_{a b d} \theta\left(k-\aleph_{k}^{c}\right) \\
& \left.+\xi\left(k-\aleph_{k}^{c}\right) \bar{D}_{a b d} \theta\left(k-\aleph_{k}^{c}\right)\right]-\theta^{T}(k)(Q-s P) \theta(k) \\
& \left.-s \theta^{T}\left(k-\aleph_{k}^{l}\right) P \theta\left(k-\aleph_{k}^{l}\right)\right\} .
\end{aligned}
$$

It follows from Lemma 2 that

$$
\begin{aligned}
& \mathbb{E}\{V(k+1)-V(k) \mid \hbar(k)\} \\
\leq & \frac{1}{s} \sum_{i, j, t=1}^{r} h_{i} h_{j} h_{t} \sum_{l=1}^{s} \mathbb{E}\left\{\left[\bar{A}_{i j t} \theta(k)+\zeta(k) \bar{B}_{j t} \theta(k)\right.\right. \\
& \left.+\bar{C}_{i j l} \theta\left(k-\aleph_{k}^{l}\right)+\xi\left(k-\aleph_{k}^{l}\right) \bar{D}_{i j l} \theta\left(k-\aleph_{k}^{l}\right)\right]^{T} Q \\
& \times\left[\bar{A}_{i j t} \theta(k)+\zeta(k) \bar{B}_{j t} \theta(k)+\bar{C}_{i j l} \theta\left(k-\aleph_{k}^{l}\right)\right. \\
& \left.+\xi\left(k-\aleph_{k}^{l}\right) \bar{D}_{i j l} \theta\left(k-\aleph_{k}^{l}\right)\right]-\theta^{T}(k)(Q-s P) \theta(k) \\
& \left.-s \theta^{T}\left(k-\aleph_{k}^{l}\right) P \theta\left(k-\aleph_{k}^{l}\right)\right\} .
\end{aligned}
$$

By noting $\mathbb{E}\left\{\zeta^{2}(k)\right\}=\sigma_{\zeta}^{2}$ and $\mathbb{E}\left\{\xi^{2}(k)\right\}=\sigma_{\xi}^{2}$, we obtain

$$
\begin{aligned}
& \mathbb{E}\{V(k+1)-V(k) \mid \hbar(k)\} \\
\leq & \frac{1}{s} \sum_{i, j, t=1}^{r} h_{i} h_{j} h_{t} \sum_{l=1}^{s} \mathbb{E}\left\{\left[\bar{A}_{i j t} \theta(k)+\bar{C}_{i j l} \theta\left(k-\aleph_{k}^{l}\right)\right]^{T}\right. \\
& \times Q\left[\bar{A}_{i j t} \theta(k)+\bar{C}_{i j l} \theta\left(k-\aleph_{k}^{l}\right)\right] \\
& -\theta^{T}(k)\left(Q-s P-\sigma_{\zeta}^{2} \bar{B}_{j t}^{T} Q \bar{B}_{j t}\right) \theta(k) \\
& -s \theta^{T}\left(k-\aleph_{k}^{l}\right) P \theta\left(k-\aleph_{k}^{l}\right) \\
& \left.+\sigma_{\xi}^{2} \theta^{T}\left(k-\aleph_{k}^{l}\right) \bar{D}_{i j l}^{T} Q \bar{D}_{i j l} \theta^{T}\left(k-\aleph_{k}^{l}\right)\right\} \\
\leq & \frac{1}{s} \sum_{i, j, t=1}^{r} h_{i} h_{j} h_{t} \sum_{l=1}^{s} \mathbb{E}\left\{\tilde{\theta}^{T}(k) \widetilde{\Omega}_{0}^{i j t l} \tilde{\theta}(k)\right\}
\end{aligned}
$$

where $\tilde{\theta}(k)=\left[\begin{array}{ll}\theta^{T}(k) & \theta^{T}\left(k-\aleph_{k}^{l}\right)\end{array}\right]^{T}$,

$$
\begin{aligned}
& \tilde{\Lambda}_{1}=-\bar{A}_{i j t}^{T} Q \bar{A}_{i j t}+Q-s P-\sigma_{\zeta}^{2} \bar{B}_{j t}^{T} Q \bar{B}_{j t}, \\
& \tilde{\Lambda}_{2}=s P-\sigma_{\xi}^{2} \bar{D}_{i j l}^{T} Q \bar{D}_{i j l}-\bar{C}_{i j l}^{T} Q \bar{C}_{i j l}, \\
& \widetilde{\Omega}_{0}^{i j t l}=\left[\begin{array}{cc}
-\tilde{\Lambda}_{1} & * \\
\bar{C}_{i j l}^{T} Q \bar{C}_{i j l} & -\tilde{\Lambda}_{2}
\end{array}\right] .
\end{aligned}
$$

Denoting

$$
\bar{\Omega}_{0}^{i j t l}=\left[\begin{array}{cccc}
-\Lambda_{1} & * & * & * \\
0 & -s P & * & * \\
0 & \sigma_{\xi} \bar{D}_{i j l} & -Q^{-1} & * \\
\bar{A}_{i j t} & \bar{C}_{i j l} & 0 & -Q^{-1}
\end{array}\right],
$$

it can be found that $\bar{\Omega}_{0}^{i j t l}$ is a principal submatrix of $\bar{\Omega}^{i j t l}$. Subsequently, $\bar{\Omega}_{0}^{i j t l}<0$ can be inferred by $\bar{\Omega}^{i j t l}<0$ in (15) which, according to the Schur Complement (Lemma 1), further implies $\widetilde{\Omega}_{0}^{i j t l}<0$. Therefore, one has

$$
\begin{aligned}
& \mathbb{E}\{V(k+1)-V(k) \mid \hbar(k)\} \\
\leq & \frac{1}{s} \sum_{i, j, t=1}^{r} h_{i} h_{j} h_{t} \sum_{l=1}^{s} \mathbb{E}\left\{\tilde{\theta}^{T}(k) \widetilde{\Omega}_{0}^{i j t l} \tilde{\theta}(k)\right\} \\
\leq & \lambda_{\max }\left(\widetilde{\Omega}_{0}^{i j t l}\right) \mathbb{E}\left\{\|\tilde{x}(k)\|^{2}\right\} \\
< & 0 .
\end{aligned}
$$

According to the definition of $V(k)$ in (16), we obtain

$$
\begin{array}{ll} 
& \lambda_{\min }(Q) \mathbb{E}\left\{\|\tilde{x}(k)\|^{2}\right\} \leq \mathbb{E}\{V(k)\} \\
\leq \quad & \lambda_{\max }(Q) \mathbb{E}\left\{\|\tilde{x}(k)\|^{2}\right\} \\
& +s \lambda_{\max }(P) \mathbb{E}\left\{\sum_{\tau=-s+1}^{-1}\|\tilde{x}(k+\tau)\|^{2}\right\} .
\end{array}
$$

For any given scalar $\mu>1$ and a sufficiently large $k$, it follows from (21) and (22) that

$$
\begin{aligned}
& \mathbb{E}\left\{\mu^{k} V(k)\right\} \\
& =\mathbb{E}\left\{V(0)+\sum_{i=0}^{k-1}\left(\mu^{i+1}(V(i+1)-V(i))\right.\right. \\
& \left.\left.+\mu^{i}(\mu-1) V(i)\right)\right\} \\
& \leq \lambda_{\max }(Q) \mathbb{E}\left\{\|\tilde{x}(0)\|^{2}\right\}+s \lambda_{\max }(P) \mathbb{E}\left\{\sum_{\tau=-s+1}^{-1}\|\tilde{x}(\tau)\|^{2}\right\} \\
& +\left(\mu \lambda_{\max }\left(\widetilde{\Omega}_{0}^{i j t l}\right)+(\mu-1) \lambda_{\max }(Q)\right) \sum_{i=0}^{k-1} \mu^{i} \mathbb{E}\left\{\|\tilde{x}(i)\|^{2}\right\} \\
& +(\mu-1) s \lambda_{\max }(P) \sum_{i=0}^{k-1} \sum_{j=-s+1}^{-1} \mu^{i} \mathbb{E}\left\{\|\tilde{x}(i+j)\|^{2}\right\} \\
& \leq \lambda_{\max }(Q) \mathbb{E}\left\{\|\tilde{x}(0)\|^{2}\right\}+s \lambda_{\max }(P) \mathbb{E}\left\{\sum_{\tau=-s+1}^{-1}\|\tilde{x}(\tau)\|^{2}\right\} \\
& +\left(\mu \lambda_{\max }\left(\widetilde{\Omega}_{0}^{i j t l}\right)+(\mu-1) \lambda_{\max }(Q)\right) \sum_{i=0}^{k-1} \mu^{i} \mathbb{E}\left\{\|\tilde{x}(i)\|^{2}\right\} \\
& +(\mu-1) s \lambda_{\max }(P)\left((s-1) \mu^{s-1} \sum_{j=-s+1}^{-1} \mathbb{E}\left\{\|\tilde{x}(j)\|^{2}\right\}\right. \\
& \left.+\left(\sum_{j=0}^{k-s} \sum_{t=1}^{s-1}+\sum_{j=k-s+1}^{k-1} \sum_{t=1}^{k-j}\right) \mu^{j} \mu^{t} \mathbb{E}\left\{\|\tilde{x}(j)\|^{2}\right\}\right) \\
& \leq \lambda_{\max }(Q) \mathbb{E}\left\{\|\tilde{x}(0)\|^{2}\right\}+s \lambda_{\max }(P) \mathbb{E}\left\{\sum_{\tau=-s+1}^{-1}\|\tilde{x}(\tau)\|^{2}\right\}
\end{aligned}
$$




$$
\begin{aligned}
& +\left(\mu \lambda_{\max }\left(\widetilde{\Omega}_{0}^{i j t l}\right)+(\mu-1) \lambda_{\max }(Q)\right) \sum_{i=0}^{k-1} \mu^{i} \mathbb{E}\left\{\|\tilde{x}(i)\|^{2}\right\} \\
& +(\mu-1) s \lambda_{\max }(P) \\
& \times\left((s-1)^{2} \mu^{s-1} \sup _{j \in \mathbb{N}[-s+1,0]} \mathbb{E}\left\{\|\tilde{x}(j)\|^{2}\right\}\right. \\
& \left.+(s-1) \mu^{s-1} \sum_{j=0}^{k-1} \mu^{j} \mathbb{E}\left\{\|\tilde{x}(j)\|^{2}\right\}\right) \\
& \leq a_{1}(\mu) \sup _{j \in \mathbb{N}[-s+1,0]} \mathbb{E}\left\{\|\tilde{x}(j)\|^{2}\right\} \\
& +a_{2}(\mu) \sum_{j=0}^{k} \mu^{j} \mathbb{E}\left\{\|\tilde{x}(j)\|^{2}\right\},
\end{aligned}
$$

where

$$
\begin{aligned}
a_{1}(\mu)= & \lambda_{\max }(Q)+s \lambda_{\max }(P)(s-1) \\
& +(\mu-1) s \lambda_{\max }(P)(s-1)^{2} \mu^{s-1}, \\
a_{2}(\mu)= & \mu \lambda_{\max }\left(\widetilde{\Omega}_{0}^{i j t l}\right)+(\mu-1) \lambda_{\max }(Q) \\
& +(\mu-1) s \lambda_{\max }(P)(s-1) \mu^{s-1} .
\end{aligned}
$$

Moreover, it is not difficult to see that $a_{1}(1)>0$ and $a_{2}(1)<$ 0 . Because $a_{1}(\mu)$ and $a_{2}(\mu)$ are continuous functions of $\mu$, we can infer that there must exist a scalar $z>1$ such that

$$
a_{1}(z)>0, \quad a_{2}(z)<0 .
$$

Subsequently, the following inequality is true

$$
\begin{aligned}
& \mathbb{E}\left\{\|\tilde{x}(k)\|^{2}\right\} \\
\leq & \sum_{j=0}^{k} \mu^{j-k} \mathbb{E}\left\{\|\tilde{x}(j)\|^{2}\right\} \\
\leq & \mu^{-k} \frac{a_{1}(z)}{-a_{2}(z)}\left(\sup _{j \in \mathbb{N}[-s+1,0]} \mathbb{E}\left\{\|\tilde{x}(j)\|^{2}\right\}-\mathbb{E}\left\{z^{k} V(k)\right\}\right) \\
\leq & \frac{a_{1}(z)}{-a_{2}(z)} \mu^{-k} \sup _{j \in \mathbb{N}[-s+1,0]} \mathbb{E}\left\{\|\tilde{x}(j)\|^{2}\right\} .
\end{aligned}
$$

By Definition 1, the augmented error dynamics (8) is exponentially stable in the mean square sense, and the proof is completed.

Now, we will show the $H_{\infty}$ disturbance attenuation performance (9) under the zero initial condition for all nonzero sequences $\{\nu(\cdot)\}$. It follows from the augmented error dynamics (8) that

$$
\begin{aligned}
& \mathbb{E}\left\{V(k+1)-V(k)+\|r(k)\|^{2}-\gamma^{2}\|v(k)\|^{2} \mid \hbar(k)\right\} \\
&= \frac{1}{s^{2}} \sum_{i, j, t, a, b, c=1}^{r} h_{i} h_{j} h_{t} h_{a} h_{b} h_{c} \sum_{l=1}^{s} \sum_{d=1}^{s} \\
& \times \mathbb{E}\left\{\left[\bar{A}_{i j t} \theta(k)+\zeta(k) \bar{B}_{j t} \theta(k)+\bar{C}_{i j l} \theta\left(k-\aleph_{k}^{l}\right)\right.\right. \\
&\left.+\xi\left(k-\aleph_{k}^{l}\right) \bar{D}_{i j l} \theta\left(k-\aleph_{k}^{l}\right)+\bar{E}_{j t} \nu(k)+\bar{F}_{i j l} \nu\left(k-\aleph_{k}^{l}\right)\right]^{T} \\
& \times Q\left[\bar{A}_{a b} \theta(k)+\zeta(k) \bar{B}_{b} \theta(k)+\bar{C}_{a b d} \theta\left(k-\aleph_{k}^{l}\right)\right. \\
&\left.+\xi\left(k-\aleph_{k}^{c}\right) \bar{D}_{a b d} \theta\left(k-\aleph_{k}^{l}\right)+\bar{E}_{b} \nu(k)+\bar{F}_{a b d} \nu\left(k-\aleph_{k}^{c}\right)\right] \\
&-\theta^{T}(k)(Q-s P) \theta(k)-s \theta^{T}\left(k-\aleph_{k}^{l}\right) P \theta\left(k-\aleph_{k}^{l}\right) \\
&+\left[\bar{G}_{i} \theta(k)+\bar{H}_{i j l} \theta\left(k-\aleph_{k}^{l}\right)+\xi\left(k-\aleph_{k}^{l}\right) \bar{J}_{i j l} \theta\left(k-\aleph_{k}^{l}\right)\right.
\end{aligned}
$$

$$
\begin{aligned}
& \left.+K \nu(k)+\bar{L}_{i j l} \nu\left(k-\aleph_{k}^{l}\right)\right]^{T}\left[\bar{G}_{a} \theta(k)+\bar{H}_{a b d} \theta\left(k-\aleph_{k}^{c}\right)\right. \\
& \left.+\xi\left(k-\aleph_{k}^{c}\right) \bar{J}_{a b d} \theta\left(k-\aleph_{k}^{c}\right)+K \nu(k)+\bar{L}_{a b d} \nu\left(k-\aleph_{k}^{c}\right)\right] \\
& \left.-\gamma^{2} \nu^{T}(k) \nu(k)-s \gamma^{2} \nu^{T}\left(k-\aleph_{k}^{l}\right) \nu\left(k-\aleph_{k}^{l}\right)\right\} \\
\leq & \frac{1}{s} \sum_{i, j, t=1}^{r} h_{i} h_{j} h_{t} \sum_{l=1}^{s} \mathbb{E}\left\{\left[\bar{A}_{i j t} \theta(k)+\bar{C}_{i j l} \theta\left(k-\aleph_{k}^{l}\right)\right.\right. \\
& \left.+\bar{E}_{j t} \nu(k)+\bar{F}_{i j l} \nu\left(k-\aleph_{k}^{l}\right)\right]^{T} Q\left[\bar{A}_{i j t} \theta(k)+\bar{C}_{i j l} \theta\left(k-\aleph_{k}^{l}\right)\right. \\
& \left.+\bar{E}_{j t} \nu(k)+\bar{F}_{i j l} \nu\left(k-\aleph_{k}^{l}\right)\right]+\left[\bar{G}_{i} \theta(k)+\bar{H}_{i j l} \theta\left(k-\aleph_{k}^{l}\right)\right. \\
& \left.+K \nu(k)+\bar{L}_{i j l} \nu\left(k-\aleph_{k}^{l}\right)\right]^{T}\left[\bar{G}_{i} \theta(k)+\bar{H}_{i j l} \theta\left(k-\aleph_{k}^{l}\right)\right. \\
& \left.+K \nu(k)+\bar{L}_{i j l} \nu\left(k-\aleph_{k}^{l}\right)\right]-s \theta^{T}\left(k-\aleph_{k}^{l}\right) P \theta\left(k-\aleph_{k}^{l}\right) \\
& +\sigma_{\xi}^{2} \theta^{T}\left(k-\aleph_{k}^{l}\right) \bar{D}_{i j l}^{T} Q \bar{D}_{i j l} \theta\left(k-\aleph_{k}^{l}\right) \\
& +\sigma_{\xi}^{2} \theta^{T}\left(k-\aleph_{k}^{l}\right) \bar{J}_{i j l}^{T} \bar{J}_{i j l} \theta\left(k-\aleph_{k}^{l}\right) \\
& -\theta^{T}(k)\left(Q-s P-\sigma_{\zeta}^{2} \bar{B}_{j t}^{T} Q \bar{B}_{j t}\right) \theta(k) \\
& \left.-\gamma^{2} \nu^{T}(k) \nu(k)-s \gamma^{2} \nu^{T}\left(k-\aleph_{k}^{l}\right) \nu\left(k-\aleph_{k}^{l}\right)\right\} .
\end{aligned}
$$

Taking advantage of the Schur Complement (Lemma 1) again, it can be seen from the matrix inequalities (15) that

$$
\begin{aligned}
& \mathbb{E}\left\{V(k+1)-V(k)+\|r(k)\|^{2}-\gamma^{2}\|v(k)\|^{2} \mid \hbar(k)\right\} \\
\leq & \frac{1}{s} \sum_{i, j, t=1}^{r} h_{i} h_{j} h_{t} \sum_{l=1}^{s} \mathbb{E}\left\{\tilde{\theta}^{T}(k) \bar{\Omega}^{i j t l} \tilde{\theta}(k)\right\} \\
< & 0,
\end{aligned}
$$

where $\tilde{\theta}(k)=\left[\begin{array}{llll}\theta^{T}(k) & \theta^{T}\left(k-\aleph_{k}^{l}\right) & \nu(k) & \nu\left(k-\aleph_{k}^{l}\right)\end{array}\right]^{T}$. By further taking the zero initial condition and $V(k) \geq 0$ into account, summing up both sides of the inequality (27) from 0 to $\infty$ yields

$$
\sum_{k=0}^{\infty} \mathbb{E}\left\{\|r(k)\|^{2}\right\} \leq \gamma^{2} \sum_{k=0}^{\infty} \mathbb{E}\left\{\|v(k)\|^{2}\right\} .
$$

According to Definition 2, the residual-generating system (3) is an $H_{\infty}$ fault detection filter, which completes the proof.

Up to now, the exponentially mean-square stability of the augmented error dynamics (8) and the $H_{\infty}$ performance have been thoroughly discussed. Nevertheless, the nonlinear term $Q^{-1}$ in $\bar{\Omega}^{i j t l}$ brings great difficulties to the semi-definite problem in Theorem 1. For this issue, we shall turn to a traceable algorithm for designing the desired fault detection filter by introducing a slack matrix variable in the next subsection.

\section{B. Synthesis of fuzzy fault detection filter}

Theorem 2. For the given structure of the fault detection filter (3), assume that there exist invertible matrix $S=$ $\operatorname{diag}\left\{S_{1}, S_{2}\right\}$, matrices $\hat{\mathscr{A}}_{i}, \hat{\mathscr{B}}_{i . l}, \hat{C}_{i}, \hat{D}_{i . l}$, and matrices $P>0$ and $Q>0$ such that the following semi-definite problem is feasible

$$
\min \gamma \quad \text { s.t. } \Omega^{i j t l}<0 \quad i, j, t \in \mathbb{U} \text { and } l \in \mathbb{S}
$$

where $\Lambda_{5}=-Q+S^{T}+S$,

$$
\Omega^{i j t l}=\left[\begin{array}{cc}
\bar{\Omega}_{1}^{i j t l} & * \\
\Omega_{2}^{i j t l} & \Omega_{3}^{i j t l}
\end{array}\right], \quad \overline{\mathscr{E}}_{j t}=\left[\begin{array}{cc}
S_{1} E_{t} & S_{1} F_{t} S_{1} B \\
-S_{2} E_{j} & -S_{2} F_{j} S_{2} B
\end{array}\right],
$$




$$
\begin{aligned}
\Omega_{2}^{i j t l} & =\left[\begin{array}{cccc}
0 & \sigma_{\xi} \overline{\mathscr{D}}_{i j l} & 0 & 0 \\
0 & \sigma_{\xi} \bar{J}_{i j l} & 0 & 0 \\
\overline{\mathscr{A}}_{i j t} & \mathscr{\mathscr { C }}_{i j l} & \overline{\mathscr{E}}_{j t} & \overline{\mathscr{F}}_{i j l} \\
\bar{G}_{i} & \bar{H}_{i j l} & K & \bar{L}_{i j l}
\end{array}\right], \\
\Omega_{3}^{i j t l} & =\operatorname{diag}\left\{-\Lambda_{5},-I_{n_{f}},-\Lambda_{5},-I_{n_{f}}\right\}, \\
\overline{\mathscr{A}}_{i j t} & =\left[\begin{array}{cc}
S_{1} A_{t} & 0 \\
\hat{\mathscr{A}}_{i}-S_{2} A_{j} & \hat{\mathscr{A}}_{i}
\end{array}\right], \quad \overline{\mathscr{C}}_{i j l}=\left[\begin{array}{cc}
0 & 0 \\
s \hat{\mathscr{B}}_{i . l} C_{j \circ l} & 0
\end{array}\right], \\
\overline{\mathscr{D}}_{i j l} & =\left[\begin{array}{cc}
0 & 0 \\
s \hat{\mathscr{B}}_{i . l} G_{j o l} & 0
\end{array}\right], \quad \overline{\mathscr{F}}_{i j l}=\left[\begin{array}{cc}
0 & 0 \\
s \hat{\mathscr{B}}_{i . l} H_{j o l} & 0
\end{array}\right] .
\end{aligned}
$$

Then, under the Round-Robin scheduling, the augmented error dynamics (8) is exponentially mean-square stable and the disturbance attenuation constraint (9) is also met. Moreover, the other gain parameters of the concerned $H_{\infty}$ fault detection filter $\hat{A}_{j}, \hat{B}_{i}$, and $\hat{D}_{i}$ are characterized by

$$
\begin{aligned}
& \hat{B}_{i}=\left[\begin{array}{lll}
S_{2}^{-1} \hat{\mathscr{B}}_{i .1} & S_{2}^{-1} \hat{\mathscr{B}}_{i .2} \cdots S_{2}^{-1} \hat{\mathscr{B}}_{i . s}
\end{array}\right] \\
& \hat{A}_{i}=S_{2}^{-1} \hat{\mathscr{A}}_{i}, \quad \hat{D}_{i}=\left[\begin{array}{lll}
\hat{D}_{i .1} & \hat{D}_{i .2} \cdots \hat{D}_{i . s}
\end{array}\right] .
\end{aligned}
$$

Proof: Noticing the relationship (30), pre- and postmultiplying both sides of $\Omega^{i j t l}<0$ with $\operatorname{diag}\left\{I_{2 n}, I_{2 n}\right.$, $\left.I_{n_{f}+n_{\omega}+n_{u}}, I_{s\left(n_{f}+n_{\omega}+n_{u}\right)}, S^{-1}, I_{2 n}, S^{-1}, I_{n_{f}}\right\}$ and its transpose yield

$$
\left[\begin{array}{ll}
\bar{\Omega}_{1}^{i j t l} & * \\
\widetilde{\Omega}_{2}^{i j t l} & \widetilde{\Omega}_{3}^{i j t l}
\end{array}\right]<0,
$$

where $Q_{s}=-S^{-1} Q S^{-T}+S^{-1}+S^{-T}, \widetilde{\Omega}_{3}^{i j t l}=$ $\operatorname{diag}\left\{-Q_{s},-I_{n_{f}},-Q_{s},-I_{n_{f}}\right\}$ and

$$
\widetilde{\Omega}_{2}^{i j t l}=\left[\begin{array}{cccc}
0 & \sigma_{\xi} \bar{D}_{i j l} & 0 & 0 \\
0 & \sigma_{\xi} \bar{J}_{i j l} & 0 & 0 \\
\bar{A}_{i j t} & \bar{C}_{i j l} & \bar{E}_{j t} & \bar{F}_{i j l} \\
\bar{G}_{i} & \bar{H}_{i j l} & K & \bar{L}_{i j l}
\end{array}\right] .
$$

On the other hand, one can infer from $Q>0$ that

$$
\begin{aligned}
Q^{-1}-Q_{s} & =Q^{-1}+S^{-1} Q S^{-T}-S^{-T}-S^{-1} \\
& =\left[S^{-1}-Q^{-1}\right] Q S^{-T}-\left[S^{-1}-Q^{-1}\right] \\
& =\left[S^{-T}-Q^{-1}\right]^{T} Q\left[S^{-T}-Q^{-1}\right] \\
& \geq 0,
\end{aligned}
$$

which means $\bar{\Omega}^{i j t l}<\Omega^{i j t l}<0$, and the proof is complete.

So far, the $H_{\infty}$ fuzzy fault detection filter has been designed to generate the desired residual signal.

Remark 3. Theorem 1 provides the sufficient conditions for the existence of the concerned fuzzy fault detection filter such that the exponentially mean-square stability of the error dynamics (8) and the optimal $H_{\infty}$ disturbance attenuation performance are met simultaneously. It should be noticed that the effects of both the multiplicative noises and the periodic communication Round-Robin protocol are reflected in the main results, and the complexity induced by the RoundRobin protocol is effectively reduced by developing a socalled block matrix method. The derivation of the desired fault detection filter is addressed in Theorem 2, where the established conditions can be verified readily by using the numerically efficient LMI toolbox in Matlab software. The residual generated by the desired fault detection filter can be used as the evaluation function (10), and the threshold (12) can be determined accordingly. Thereafter, the possible fault in the networked fuzzy system can be detected according to the rule (13). On the other hand, it can be seen from (2) and (3) that the time-varying delay $\aleph_{k}^{l}$ is a key factor to influence the performance of the fault detection filter. Therefore, we know from $\aleph_{k}^{l}=\bmod (k-l, s)$ that the dimension of the measured output vector $y(k)$ and the measurement vector $\vec{y}(k)$ can greatly affect fault detection result. As discussed previously, the designed adaptive threshold is superior to the traditional constant one since the current threshold can be adjusted by shifting the control input vector $u(k)$.

\section{ILLuSTRATIVE EXAMPLE}

In this section, we present a numerical example to demonstrate the feasibility of the proposed algorithm. Consider a discrete-time networked fuzzy system (1) with three measurement sensors (i.e. $s=3$ ). Take the number of IF-THEN rules $r=2$ and the other parameters as follows:

$$
\begin{aligned}
A_{1} & =\left[\begin{array}{ll}
-0.4223 & -0.1022 \\
-0.0235 & -0.1002
\end{array}\right], D_{2}=\left[\begin{array}{ll}
0.3841 & 0.2671 \\
0.0323 & 0.0154
\end{array}\right], \\
E_{1} & =\left[\begin{array}{ll}
-0.4102 \\
-0.3205
\end{array}\right], F_{1}=\left[\begin{array}{l}
-0.4036 \\
-0.0085
\end{array}\right], B_{1}=\left[\begin{array}{c}
0.6231 \\
-0.3251
\end{array}\right], \\
A_{2} & =\left[\begin{array}{ll}
0.3125 & 0.1035 \\
0.3231 & 0.1705
\end{array}\right], D_{1}=\left[\begin{array}{ll}
-0.2334 & -0.1253 \\
-0.4869 & -0.0254
\end{array}\right], \\
E_{2} & =\left[\begin{array}{cc}
0.0065 \\
-0.4008
\end{array}\right], F_{2}=\left[\begin{array}{cc}
-0.5045 \\
0.1007
\end{array}\right], B_{2}=\left[\begin{array}{c}
-06423 \\
-0.3251
\end{array}\right], \\
C_{1} & =\left[\begin{array}{cc}
-0.3152 & -0.4523 \\
-0.0561 & -0.1357 \\
-0.0025 & 0.0058
\end{array}\right], H_{1}=\left[\begin{array}{l}
-0.0254 \\
-0.3251 \\
-0.0005
\end{array}\right], \\
G_{1} & =\left[\begin{array}{cc}
-0.4058 & 0.0169 \\
0.2363 & -0.2895 \\
0.1004 & 0.1710
\end{array}\right], H_{2}=\left[\begin{array}{ll}
0.2854 \\
0.0304 \\
0.3588
\end{array}\right], \\
C_{2} & =\left[\begin{array}{cc}
0.0085 & -0.1006 \\
-0.1036 & -0.0025 \\
0.0552 & 0.1035
\end{array}\right], G_{2}=\left[\begin{array}{ll}
0.2221 & 0.4016 \\
0.0059 & 0.0336 \\
0.1235 & 0.0410
\end{array}\right] .
\end{aligned}
$$

The variances of the zero-mean multiplicative noises $\zeta(k)$ and $\xi(k)$ are set to be $\sigma_{\zeta}=1.1$ and $\sigma_{\xi}=1.4$, respectively.

By resorting to the solver of mincx in LMI toolbox, the convex optimization problem (29) is solved and the $H_{\infty}$ performance index is optimized as $\gamma_{\min }=1.1409$, where the feasible solutions of the positive definite matrices $Q$ and $P$ as well as the invertible matrices $S_{1}$ and $S_{2}$ are listed below:

$$
\begin{aligned}
P & =\left[\begin{array}{cccc}
0.03330 & -0.00322 & 0 & 0 \\
-0.00322 & 0.05446 & 0 & 0 \\
0 & 0 & 0.00006 & -0.00004 \\
0 & 0 & -0.00004 & 0.00013
\end{array}\right], \\
Q & =\left[\begin{array}{cccc}
0.33648 & 0.06371 & -0.00411 & 0.00003 \\
0.06371 & 0.23834 & 0.00003 & -0.00124 \\
-0.00411 & 0.00003 & 0.01148 & 0.00724 \\
0.00003 & -0.00124 & 0.00724 & 0.01809
\end{array}\right], \\
S_{1} & =\left[\begin{array}{lll}
0.28780 & 0.04682 \\
0.04683 & 0.17911
\end{array}\right], \quad S_{2}=\left[\begin{array}{ll}
0.06347 & 0.14937 \\
0.14937 & 0.41285
\end{array}\right] .
\end{aligned}
$$


Subsequently, the gain matrices of the concerned fault detection filter are obtained as follows:

$$
\begin{aligned}
& \hat{A}_{1}=\left[\begin{array}{cc}
-0.2882 & 0.1503 \\
-0.0590 & -0.2604
\end{array}\right], \quad \hat{A}_{2}=\left[\begin{array}{cc}
0.0557 & 0.2925 \\
0.0080 & -0.0742
\end{array}\right] \text {, } \\
& \hat{B}_{1}=\left[\begin{array}{lll}
-0.0865 & -0.1572 & -0.2684 \\
-0.1688 & -0.3580 & -0.6317
\end{array}\right] \text {, } \\
& \hat{B}_{2}=\left[\begin{array}{ccc}
0.0134 & -0.1674 & -0.1674 \\
-0.2845 & -0.3631 & -0.3631
\end{array}\right] \text {, } \\
& \hat{C}_{1}=\left[\begin{array}{ll}
0.0082 & 0.0060
\end{array}\right], \quad \hat{C}_{2}=\left[\begin{array}{ll}
0.01093 & -0.0053
\end{array}\right] \text {, } \\
& \hat{D}_{1}=\left[\begin{array}{lll}
-0.0477 & -0.1354 & -0.2734
\end{array}\right] \text {, } \\
& \hat{D}_{2}=\left[\begin{array}{lll}
-0.0296 & -0.1272 & -0.1660
\end{array}\right] \text {. }
\end{aligned}
$$

By now, an $H_{\infty}$ fault detection filter has been designed under the framework of the T-S fuzzy model.

For simulation purpose, we adopt the normalized membership functions as

$$
h_{1}=\frac{\sin ^{2}\left(x_{1}(k)\right)}{2+\cos \left(x_{1}(k)\right)}, \quad h_{2}=1-\frac{\sin ^{2}\left(x_{1}(k)\right)}{2+\cos \left(x_{1}(k)\right)} .
$$

The multiplicative noises are taken as

$$
\zeta(k)=-10^{-\operatorname{tg}^{-1}(k)} \frac{\sin (4 k)}{\sin (k)}\left(\operatorname{tg}^{-1}(k)\right)^{2} \varepsilon(k)
$$

with $\varepsilon(k)$ being uniformly distributed over $[0,1]$, and $\xi(k)=\operatorname{tg}^{-1}(k) \zeta(k)$. Let the control input be $u(k)=$ $0.12 e^{\sin \left(k \operatorname{tg}^{-1}(k)\right)} \operatorname{tg}^{-1}(k \cos (k))$, the disturbance input be $\omega(k)=e^{-10 k}$, the initial state be $\phi(k)=0\left(\forall k \in \mathbb{Z}^{-}\right)$, the evaluating time horizon be $\mathcal{L}=15$, and the fault be

$$
f(k)=\left\{\begin{array}{cc}
0.05 k e^{\frac{-k}{80}}, & 30 \leq k \leq 120 \\
0, & \text { else }
\end{array}\right.
$$

By using the designed fault detection filter (3), the residual signal is steadily generated once the sensor measurements are communicated to the fault detection filter. Fig. 3 shows the evolutions of the residual with fault and fault free, which indicates that the residual will appear even if the fault does not occur. When the fault arises in the fuzzy system, the curve of residual signal yields a surge, and it begins to flatten when the fault disappeared. Evaluation of the residual is shown in Fig. 4, where it can be seen that the growth of the residual evaluation function starts to slacken when the fault disappears in the target networked fuzzy systems.

With help of the designed residual evaluation function (10), the threshold is determined as $J_{t h}=0.0636$ after 100 runs of Monte Carlo simulations. By checking the values of the residual evaluation function $J(k)$, we find that $J(k)=0.0666$ exceeds the threshold at the time instant $k=37$ for the first time, and the alarm apparatus can thus be triggered, which means that the fault in the target system is successfully detected with only 7 time instants behind. The evaluation of the residual without fuzzy rules is shown in Fig. 5, from which one knows $J(k)=0.0647$ exceeds the threshold when $k=61$. In other words, the non-fuzzy fault detection result largely lags behind the fault occurrence, which thus demonstrates the superiority of the studied scheme.

On the other hand, the measurement model (2) implies that the time delays induced by the Round-Robin protocol

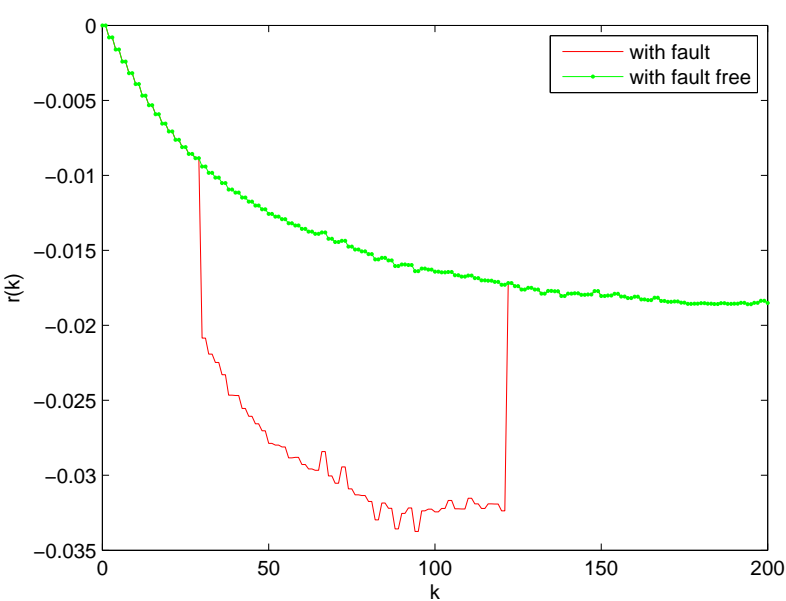

Fig. 3: Residual signal

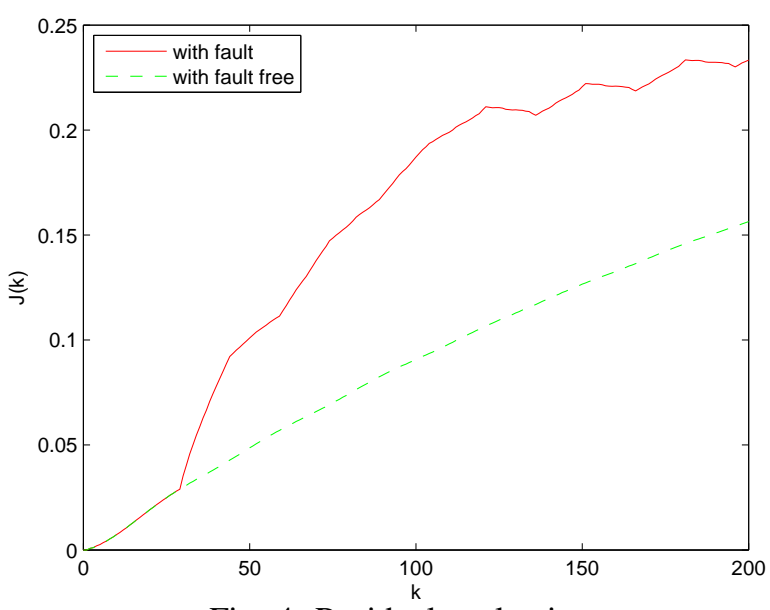

Fig. 4: Residual evaluation

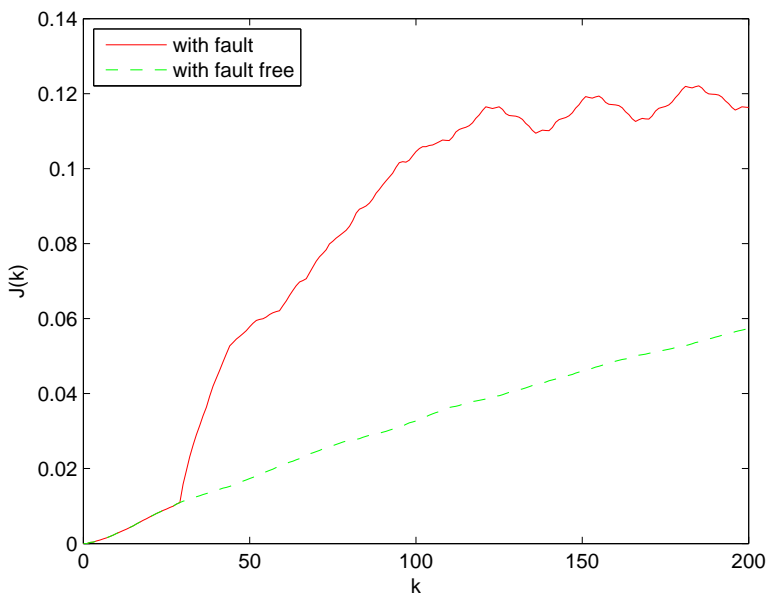

Fig. 5: Residual evaluation without fuzzy rules

vary periodically. Trajectories of the residual with and without the multiplicative noises and interference input are shown in Fig. 6, from which we can see that the multiplicative noises $\zeta(k)$ and $\xi(k)$, the interference $\omega(k)$ can all produce a dash to the residual signal. 


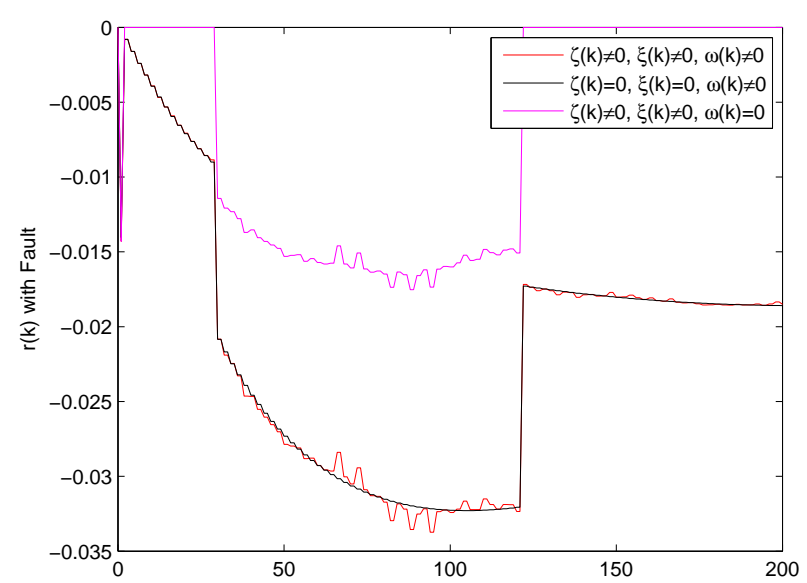

Fig. 6: Impact of the multiplicative noises and disturbance input to the residual

\section{Conclusions}

The fuzzy fault detection problem has been addressed for a class of T-S fuzzy networked systems in this paper. A novel communication scheduling solution has been used to deal with the communication constraints and the $\mathrm{ZOHs}$ have been used to improve the utilization of the received sensor measurements. In the framework of T-S fuzzy model, the structure of an $H_{\infty}$ fuzzy fault detection filter embedded in the concerned fault detector has been suggested. The explicit expression of the desired fault detection filter has been characterized by solutions to a set of LMI constraints. To efficiently detect the fault occurred in the networked fuzzy systems, a finitetime residual evaluation function and the threshold consisting of constant and adjustable parts have been designed. On the other hand, the low requirement on bandwidth under RoundRobin protocol makes it can be widely used to some special applications such as the fault detection of the underwater power system, the forest fire monitoring and the remote medical diagnosis, etc. Moreover, for energy saving purpose, the event-triggered strategy can play an important role in the low-power network communication, see. e.g. [23]. Therefore, the above topics under Round-Robin protocol will constitute our further research directions.

\section{REFERENCES}

[1] D. Agarwal and N. Kishor, A fuzzy inference-based fault detection scheme using adaptive thresholds for health monitoring of offshore wind-farms, IEEE sensors journal, Vol. 14, No. 11, pp. 3851-3861, 2014.

[2] S. Amin, G. A. Schwartz, and S. S. Sastry, Security of interdependent and identical networked control systems, Automatica, Vol. 49, No. 1, pp. 186-192, 2013.

[3] S. Boyd, L. E. Ghaoui, E. Feron, and V. Balakrishnan, Linear Matrix Inequalities in Systems and Control Theory. Philadelphia: Society for Industrial and Applied Mathematics (SIAM), USA, 1994.

[4] M. Chadli and H. R. Karimi, Robust observer design for unknown inputs Takagi-Sugeno models, IEEE Transactions on Fuzzy Systems, Vol. 21, No. 1, pp. 158-164, 2013.

[5] D. Ding, Z. Wang, D. W. C. Ho and G. Wei, Observer-based eventtriggering consensus control for multiagent systems with lossy sensors and cyber-attacks, IEEE Transactions on Cybernetics, vol. 47, no. 8, pp. 1936-1947, Aug. 2017.
[6] D. Ding, Z. Wang, D. W. C. Ho and G. Wei, Distributed recursive filtering for stochastic systems under uniform quantizations and deception attacks through sensor networks, Automatica, vol. 78, pp. 231-240, Apr. 2017.

[7] H. Dong, Z. Wang, and H. Gao, Robust filtering for a class of nonlinear networked systems with multiple stochastic communication delays and packet dropouts, IEEE Transactions on Signal Processing, Vol. 58, No. 4, pp. 1957-1966, 2010.

[8] H. Dong, Z. Wang, J. Lam, and H. Gao, Fuzzy-model-based robust fault detection with stochastic mixed time-delays and successive packet dropouts, IEEE Transactions on Systems, Man, and Cybernetics-Part B, Vol. 42, No. 2, pp. 365-376, 2012.

[9] X. Guan and C. Chen, Delay-dependent guaranteed cost control for T-S fuzzy systems with time delays, IEEE Transactions on Fuzzy Systems, Vol. 12, No. 2, pp. 236-249, 2004.

[10] F. Hsiao, C. Chen, Y. Liang, S. Xu, and W. Chiang, T-S fuzzy controllers for nonlinear interconnected systems with multiple time delays, IEEE Transactions on Circuits and Systems I: Regular Papers, Vol. 52, No. 9, pp. 1883-1893, 2005.

[11] D. Hristu and K. Morgansen, Limited communication control, Systems \& Control Letters, Vol. 37, No. 4, pp. 193-205, 1999.

[12] Y. Halevi and A. Ray, Integrated communication and control systems: Part I-Analysis, Journal of Dynamic Systems, Measurement, and Control, Vol. 110, No. 4, pp. 367-373, 1988

[13] X. He, Z. Wang, Y. Ji, and D. Zhou, Robust fault detection for networked systems with distributed sensors, IEEE Transactions on Aerospace and Electronic Systems, Vol. 47, No. 1, pp. 166-177, 2011.

[14] X. He, Z. Wang, and D. Zhou, Robust fault detection for networked systems with communication delay and data missing, Automatica, Vol. 46, No. 11, pp. 2634-2639, 2009.

[15] J. Li, H. Dong, F. Han, N. Hou and X. Li, Filter design, fault estimation and reliable control for networked time-varying systems: A survey, Systems Science \& Control Engineering: An Open Access Journal, vol. 5, pp. 331-341, 2017.

[16] L. Li, S. X. Ding, J. Qiu, Y. Yang and Y. Zhang, Weighted fuzzy observer-based fault detection approach for discrete-time nonlinear systems via piecewise-fuzzy Lyapunov functions, IEEE Transactions on Fuzzy Systems, Vol. 24, No. 6, pp. 1320-1333, Dec. 2016.

[17] J. Lian, J. Liu, and Y. Zhuang, Mean stability of positive Markov jump linear systems with homogeneous and switching transition probabilities, IEEE Transactions on Circuits and Systems-II: Express Briefs, vol. 62, no. 8, pp. 801-805, 2015.

[18] K. Liu, E. Fridman, and L. Hetel, Stability and $L_{2}$-gain analysis of networked control systems under Round-Robin scheduling: a time-delay approach, Systems \& Control Letters, Vol. 61, No.5, pp. 666-675, 2012.

[19] F. Li, P. Shi, C. C. Lim, and L. Wu, Fault detection filtering for nonhomogeneous Markovian jump systems via fuzzy approach, IEEE Transactions on Fuzzy Systems, 2016. DOI: 10.1109/TFUZZ.2016.2641022.

[20] Y. Liu, Z. Wang, X. He, and D. Zhou, Filtering and fault detection for nonlinear systems with polynomial approximation, Automatica, Vol. 54, pp. 348-359, 2015.

[21] J. Liang, Z. Wang, B. Shen, and X. Liu, Distributed state estimation in sensor networks with randomly occurring nonlinearities subject to time delays, ACM Transactions on Sensor Networks (TOSN), Vol. 9, No. 1, Article No. 4, 2012.

[22] L. Ma, Z. Wang, Q.-L. Han and H. K. Lam, Variance-constrained distributed filtering for time-varying systems with multiplicative noises and deception attacks over sensor networks, IEEE Sensors Journal, Vol. 17, No. 7, pp. 2279-2288, Apr. 2017.

[23] L. Ma, Z. Wang, and H. K. Lam, Event-triggered mean-square consensus control for time-varying stochastic multi-agent system with sensor saturations, IEEE Transactions on Automatic Control, Vol. 62, No. 7, pp. 3524-3531, 2017.

[24] Z. Mao, Y. Zhan, G. Tao, B. Jiang, and X.-G. Yan, Sensor fault detection for rail vehicle suspension systems with disturbances and stochastic noises, IEEE Transactions on Vehicular Technology, vol. 66, no. 6, pp. 4691-4705, 2017.

[25] Z. Mao, Y. Wang, B. Jiang, and G. Tao, Fault diagnosis for a class of active suspension systems with dynamic actuators' faults, International Journal of Control, Automation and Systems, vol. 14, no.5, pp. 11601172, 2016.

[26] Y. Niu, D. W. C. Ho and C. W. Li, Filtering for discrete fuzzy stochastic systems with sensor nonlinearities, IEEE Transactions on Fuzzy Systems, Vol. 18, No. 5, pp. 971-978, 2010.

[27] J. Qiu, G. Feng, and H. Gao, Asynchronous output-feedback control of networked nonlinear systems with multiple packet dropouts: T-S fuzzy 
affine model-based approach, IEEE Transactions on Fuzzy Systems, Vol. 19, No. 6, pp. 1014-1030, 2011.

[28] B. J. Rhee and S. Won, A new fuzzy Lyapunov function approach for a Takagi-Sugeno fuzzy control system design, Fuzzy sets and systems, Vol. 157, No. 9, pp. 1211-1228, 2006.

[29] Q. Shen, B. Jiang and P. Shi, Adaptive fault diagnosis for T-S fuzzy systems with sensor faults and system performance analysis, IEEE Transactions on Cybernetics, Vol. 22, No. 2, pp. 274-285, 2014.

[30] L. Shi, P. Cheng, and J. Chen, Sensor scheduling with limited communication energy and bandwidth, in Proceedings of 2010 11th International Conference on Control Automation Robotics \& Vision (ICARCV), pp. 537-542, Singapore, 7-10th December 2010.

[31] P. Subbaraj and B. Kannapiran, Fault detection and diagnosis of pneumatic valve using adaptive neuro-fuzzy inference system approach, Applied Soft Computing, Vol. 19, pp. 362-371, 2014.

[32] M. Shahriari-kahkeshi and F. Sheikholeslam, Adaptive fuzzy wavelet network for robust fault detection and diagnosis in non-linear systems, IET Control Theory \& Applications, Vol. 8, No. 15, pp. 1487-1498, 2014.

[33] F. Sun, J. Liang, and W. Gong, $H_{\infty}$ filtering for time-varying delayed systems with randomly varying sensor saturations, in Proceedings of 33 rd Chinese Control Conference (CCC), pp. 5247-52, Nanjing, China, 28-30th July 2014.

[34] C. S. Tseng, B. S. Chen, and H. J. Uang, Fuzzy tracking control design for nonlinear dynamic systems via T-S fuzzy model, IEEE Transactions on Fuzzy Systems, Vol. 9, No. 3, pp. 381-392, 2001.

[35] V. Ugrinovskii and E. Fridman, A Round-Robin type protocol for distributed estimation with $H_{\infty}$ consensus, Systems \& Control Letters, Vol. 69, pp. 103-110, 2014.

[36] D. Wang, J. Wang, and W. Wang, $H_{\infty}$ controller design of networked control systems with Markov packet dropouts, IEEE Transactions on Systems, Man and Cybernetics: Systems, vol. 43, no. 3, pp. 689-697, 2013.

[37] Z. Wang, D. W. Ho, and X. Liu, A note on the robust stability of uncertain stochastic fuzzy systems with time-delays, IEEE Transactions on Systems, Man and Cybernetics, Part A: Systems and Humans, Vol. 34 No. 4, pp. 570-576, 2004.

[38] W. S. Wong and R. W. Brockett, Systems with finite communication bandwidth constraints-II: Stabilization with limited information feedback, IEEE Transactions on Automatic Control, Vol. 44, No. 5, pp. 10491053, 1999.

[39] T. Wu, F. Li, C. Yang, and W. Gui, Event-based fault detection filtering for complex networked jump systems, IEEE/ASME Transactions on Mechatronics, 2017. DOI: 10.1109/TMECH.2017.2707389.

[40] Z. Wang, F. Yang, D. W. Ho, and X. Liu, Robust $H_{\infty}$ control for networked systems with random packet losses, IEEE Transactions on Systems, Man, and Cybernetics, Part B: Cybernetics, Vol. 37, No. 4 pp. 916-924, 2007.

[41] Z. Wang, D. M. Anand, J. Moyne and D. M. Tilbury, Improved sensor fault detection, isolation, and mitigation using multiple observers approach, Systems Science \& Control Engineering: An Open Access Journal, Vol. 5, No. 1, 2017, pp. 70-96.

[42] F. Yang, Z. Wang, and Y. Hung, Robust Kalman filtering for discrete time-varying uncertain systems with multiplicative noises, IEEE Transactions on Automatic Control, Vol. 47, No. 7, pp. 1179-1183, 2002.

[43] L. Zhang and D. Hristu-Varsakelis, Communication and control codesign for networked control systems, Automatica, Vol. 42, No. 6, pp. 953-958, 2006.

[44] S. Zhang, Z. Wang, D. Ding, H. Shu, T. Hayat, and A. M. Dobaie, On design of fault detection filter in finite frequency domain with regional pole assignment, IEEE Transactions on Circuits and Systems-Part II, Vol. 62, No. 4, pp. 382-386, 2015.

[45] M. Zhong, Y. Song, S. X. Ding. Parity space-based fault detection for linear discrete time-varying systems with unknown input, Automatica, Vol. 59, pp. 120-126, 2015.

[46] M. Zhong, S. X. Ding, and D. Zhou. A New Scheme of Fault Detection for Linear Discrete Time-varying Systems, IEEE Transactions on Automatic Control, Vol. 61, No. 9, pp. 2597-2602, 2016.

[47] M. Zhong, S. X. Ding, J. Lam, and H. Wang, An LMI approach to design robust fault detection filter for uncertain LTI systems, Automatica, Vol. 39, No. 3, pp. 543-550, 2003.

[48] X. Zhu and Y. Xia, $H_{\infty}$ descriptor fault detection filter design for T-S fuzzy discrete-time systems, Journal of the Franklin Institute, Vol. 351, No. 12, pp. 5358-5375, 2014.

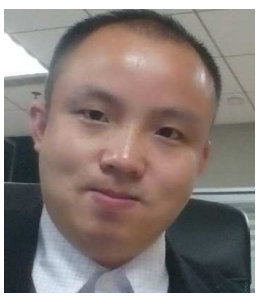

Yuqiang Luo was born in Guizhou Province, China, in 1985. He received the B.Sc. degree in mechanism design, manufacturing and automatization and the M.Sc. degree in control engineering from University of Shanghai for Science and Technology, Shanghai, China, in 2009 and 2015, respectively. He is now a $\mathrm{Ph} . \mathrm{D}$. candidate in the School of Optical-Electrical and Computer Engineering at University of Shanghai for Science and Technology, Shanghai, China. He is also an assistant engineer in the network center of the university.

His current research interests include nonlinear stochastic control and filtering theory, multidimensional systems and network communication systems. $\mathrm{He}$ is a very active reviewer for many international journals.

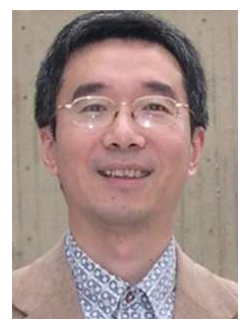

Zidong Wang (SM'03-F'14) was born in Jiangsu, China, in 1966. He received the B.Sc. degree in mathematics from Suzhou University, Suzhou, China, in 1986, and the M.Sc. degree in applied mathematics and the Ph.D. degree in electrical engineering from the Nanjing University of Science and Technology, Nanjing, China, in 1990 and 1994, respectively.

He is currently a Professor of dynamical systems and computing with the Department of Information Systems and Computing, Brunel University London, Uxbridge, U.K. From 1990 to 2002, he held teaching and research appointments in universities in China, Germany, and the U.K. He has published over 300 papers in refereed international journals. His current research interests include dynamical systems, signal processing, bioinformatics, control theory, and applications.

Prof. Wang was a recipient of the Alexander von Humboldt Research Fellowship of Germany, the JSPS Research Fellowship of Japan, and the William Mong Visiting Research Fellowship of Hong Kong. He serves (or has served) as the Editor-in-Chief of Neurocomputing, and an Associate Editor for 12 international journals, including the IEEE TRANSACTIONS ON AUTOMATIC CONTROL, the IEEE TRANSACTIONS ON CONTROL SYSTEMS TECHNOLOGY, the IEEE TRANSACTIONS ON NEURAL NETWORKS, the IEEE TRANSACTIONS ON SIGNAL PROCESSING, and the IEEE TRANSACTIONS ON SYSTEMS, MAN, AND CYBERNETICSPART C: APPLICATIONS AND REVIEWS. He is a Fellow of the Royal Statistical Society and a member of program committee for several international conferences.

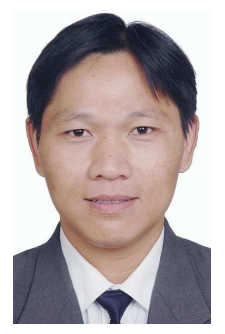

Guoliang Wei received the B.Sc. degree in mathematics from Henan Normal University, Xinxiang, China, in 1997 and the M.Sc. degree in applied mathematics and the Ph.D. degree in control engineering, both from Donghua University, Shanghai, China, in 2005 and 2008, respectively. He is currently a Professor with the Department of Control Science and Engineering, University of Shanghai for Science and Technology, Shanghai, China.

From March 2010 to May 2011, he was an Alexander von Humboldt Research Fellow in the Institute for Automatic Control and Complex Systems, University of DuisburgEssen, Germany. From March 2009 to February 2010, he was a post doctoral research fellow in the Department of Information Systems and Computing, Brunel University, Uxbridge, UK, sponsored by the Leverhulme Trust of the UK From June to August 2007, he was a Research Assistant at the University of HongKong. From March to May 2008, he was a Research Assistant at the City University of Hong Kong.

His research interests include nonlinear systems, stochastic systems, and bioinformatics. He has published more than 50 papers in refereed international journals. He is a very active reviewer for many international journals. 